\title{
Boey Score in Predicting Outcome in Perforated Peptic Ulcer from Tertiary Referral Center of Nepal
}

\author{
Sah Dhruba Narayan MBBS, MS, MCh* \\ Surgical Gastroenterology, Resident Institute of Medicine, TU Teaching Hospital, Kathmandu, Nepal \\ *Corresponding Author: Sah Dhruba Narayan, Dept. of GI \& General Surgery, Maharajgunj, Kathmandu, \\ Nepal,Email: drshah_dn@live.com
}

\begin{abstract}
Background: Accurate and early identification of Perforated Peptic Ulcer (PPU) patients with an increased risk of adverse outcome is needed to plan and target the level of perioperative monitoring and treatment. The goal of the study is to determine the accuracy of the Boey scoring system in predicting mortality and morbidity in PPU patients in comparison to other outcome predictors.
\end{abstract}

Methods: This is a prospective observational study conducted at BIR hospital, Nepal from October 2014 January 2016. A total of 50 cases undergoing emergency surgery for PPU were included. Receiver-operating characteristic curve analysis was used to compare predictive ability between Boey score and other outcome predictors.

Results: 30-days mortality was $18 \%$ while postoperative morbidity recorded in $64 \%$. ROC curve analysis revealed Boey score area under curve (AUC) mortality prediction (0.802); morbidity (0.778). Patients with morbidity and mortality had statistically significant higher mean \pm S.D value of Boey score. Besides, Boey score, Peptic Ulcer Perforation (PULP) score predicted much better.

Conclusions: Early presentation, prompt diagnosis and emergent surgery are the pillars to successful management and good outcomes of PPU. The Boey score serve as a simple and precise predictor of postoperative mortality and morbidity.

Keywords: Perforated peptic ulcer, Scoring, Boey, PULP, Outcomes, NAMS

\section{INTRODUCTION}

Peptic ulcer disease (PUD) affects 4 million people around the world yearly out of which $10 \%-20 \%$ of these patients develop complications and $2 \%-14 \%$ of the ulcers will perforate (1). Mortality and morbidity following perforated peptic ulcer (PPU) is substantial, and mortality rates of $25-30 \%$ have been reported indifferent published studies $(2,3)$. Surgery is most successful mode of treatment of perforated peptic ulcer peritonitis. Formal acid-reducing procedures like vagotomy with or without drainage procedures, which has historically been the mainstay of PPU therapy, are now being replaced by simpler procedures, such as omental patch primary closure of the perforation, owing to better understanding of the pathophysiology of peptic ulcer and successful eradication of $\mathrm{H}$. pylori treatment. Perforated duodenal ulcer treated by patch repair with a vascularized omental pedicle commonly referred to as a Graham patch or omentopexy. However, in cases of perforated gastric ulcer, either ulcer excision and repair of the defect or biopsy and omental patch are the most expeditious approach in the emergency setting.

Poor outcomes in PPU have been associated with increasing age, major medical illness, perioperative hypotension and delay in diagnosis and management(4). Surgical repair should not be delayed in patients with general peritonitis because every hour of delay increases the mortality risk (5). Stratification of patients into different categories based on the likelihood of morbidity and mortality is important to facilitate management of PPU patients and to improve the outcomes, so that high-risk patients can receive more appropriate treatment and better intensive care. Appropriate risk assessment and selection of therapeutic alternatives becomes important to address the risk for morbidity and mortality. Various risk scores in PPU patients have been developed for the prediction of outcomes (6-9). The most relevant prediction rule for PPU is the 
Boey score (7)which seeks to predict mortality based on the presence of major medical illness, preoperative shock, and perforation longer than $24 \mathrm{hr}$. In the original study by Boeyet al., the inhospital mortality proportion increased progressively with the number of prognostic variables, being $0 \%, 10 \%, 45.5 \%$, and $100 \%$ in patients with none, one, two, or all three variables, respectively. The Boey score has been re-evaluated in a number of relatively small and single center studies, but neither Irvin(10), Lee et al.(8), Chandra and Kumar (11), Makela et al. (9), nor Lohsiriwat et al. (12) could fully replicate the convincing results found by Boey et al. The Boeyscore is simple to calculate, consisting of only three parameters and was created specifically for patients with PPU. The accuracy rate in predicting mortality was $93.9 \%$ and there were no false negative errors (7). The Boey scoring system (7) is among the common risk stratifications used because of its simplicity and high predictive value for mortality of PPU patients $(6,9)$. However, its accuracy in predicting morbidity is still questionable(8). Peptic Ulcer Perforation (PULP) score (13) includes seven factors with weighted points applicable for each factor, with a maximum sum of 18 points being the highest possible. The most commonly used preoperative clinical prediction rule worldwide is the American Society of Anesthesiologists (ASA) score(14, 15). In the ASA scoring system, the patient's preoperative health status, independent of the current surgical disease, is graded in five categories. The Mannheim Peritonitis Index (MPI) $(16,17)$ has scores from 0 to 47 with eight adverse factors, comprising both preoperative and perioperative conditions.

The aim of the study is to compare the accuracy of Boey scoring systems in predicting mortality and morbidity in PPU patients in comparison to other scoring systems like PULP, ASA and MPI.

\section{METHODS}

This was a Prospective observational/ noninterventional study conducted at Department of Surgery, National Academy of Medical Sciences (NAMS), Bir Hospital, Kathmandu, Nepal from October2014 to January 2016 for 16 months' duration. Inclusion criteria were all perforated peptic ulcer with age $\geq 16$ years with clinically \& radiologically suspected cases of PPU and/ or intraoperative diagnosed cases of perforation over duodenum and stomach. Exclusion criteria were perforation owing to trauma, non-surgically treated patientsor unfit for general anesthesia, not giving consent, patient follow up criteria not met. Ethical approval taken from Institutional Review Board (IRB, NAMS). Definitive diagnosis was made on explorative laparotomy by finding duodenal/ gastric perforation. The strategy of PPU treatment in our hospital was to perform primary closure of the perforation with classical or modified Graham's patch omentopexy. Gastric resection with gastrojejunostomy or gastrojejunostomy with primary repair was performed in the case of perforated proximal gastric ulcer or large perforated ulcer (diameter $>20 \mathrm{~mm}$ ), depending on the surgeon's intra operative decision. Each patients risk score had been categorized in Boey, PULP, ASA, MPI scoring system. Shock was defined as systolic BP of less than $90 \mathrm{~mm} \mathrm{Hg}$ at presentation to emergency and duration of perforation was defined as duration between onset of pain abdomen and presentation to emergency. Standard postoperative care given along with $\mathrm{H}$. pylori eradication with a 2-weeks triple-drug therapy followed by 4 weeks PPI. Patients were followed-up for 30 days after surgeryfor any complications. Any significant perioperative event was noted and managed as per institutional practice.

Wound infections were managed with antibiotics as per culture \& sensitivity report along with lay it opens and secondary suturing as per individual basis. Similarly, other postoperative complications were managed as per institutional practice.

The data were analyzed using SPSS version 23. A $95 \%$ confidence interval was taken, and $\mathrm{p}$ value less than 0.05 was termed as statistically significant. Receiver-operating characteristic (ROC) curve analysis were used to estimate the predictive ability of the Boey score in assessing the postoperative morbidity and mortality and compared with ASA, MPI, and PULP score.

\section{RESULTS}

A total of 50 operated patients for PPU were included in the study with male predominance (92\%) and mean \pm SD age of $45.16 \pm 16.65$ years. Alcohol, smoking and history of PUD were the notable risk factors. Twenty six percentages of patients had comorbidities out of which diabetes mellites (DM) were most common followed by cardiac diseases. Median duration of perforation was 30 hours (range, 7360 hours). Perforation duration of $>24$ hours were present in 29 patients while 10 patients 
presented with $>48$ hours. Shocks at admission were present in 11 cases. First part of duodenum over anterior aspect was the most common (41) site of perforation besides gastric (antrum/ body -5) and Juxtapyloric (4). Median size of perforation was $6.5 \mathrm{~mm}$ (range, 3-25 mm). Clear peritoneal exudate were present in 21 cases while cloudly purulent in 29 cases. The average time of operation were $69.92 \pm 27.34 \mathrm{~min}$. Majority $(86 \%)$ patients were operated with classical Grahms' patch omentopexy while remaining procedures were modified Grahms' patch omentopexy (2), omentopexy with gastrojejunostomy (2), peritoneal lavage (1), subtotal gastrectomy (1), primary repair \& feeding jejunostomy (1). Categorizing patients as per different scoring systems (mean \pm S.D), Boey score had $1.04 \pm 1.01$ while others were PULP $(3.88 \pm 3.37)$, ASA $(2.24 \pm 1.29)$ and MPI (16.26 \pm 8.19). Regarding 30 days outcomes, total of 9 mortality occurred and the causes were ARDS (5), Refractory Septic Shock (1), Cardiac Failure (1), Renal Failure (1), Others (1). Similarly, $64 \%$ of patients developed complications out of which $30 \%$ had Clavien - Dindo grade III \& above. Median hospital stay was 8 days (IQR, 6-11 days). In table1, outcomes of individual Boey score of all the operated patients have been shown.

Table1. Boey Score and Outcomes (number of patients /\%)

\begin{tabular}{|l|l|l|l|}
\hline $\begin{array}{l}\text { Risk } \\
\text { Score }\end{array}$ & $\begin{array}{l}\text { Number of } \\
\text { patients }\end{array}$ & Morbidity & Mortality \\
\hline 0 & 19 & $8(42 \%)$ & $1(5.3 \%)$ \\
\hline 1 & 15 & $8(53 \%)$ & $1(6.7 \%)$ \\
\hline 2 & 11 & $11(100 \%)$ & $4(36.4 \%)$ \\
\hline 3 & 5 & $5(100 \%)$ & $3(60 \%)$ \\
\hline
\end{tabular}

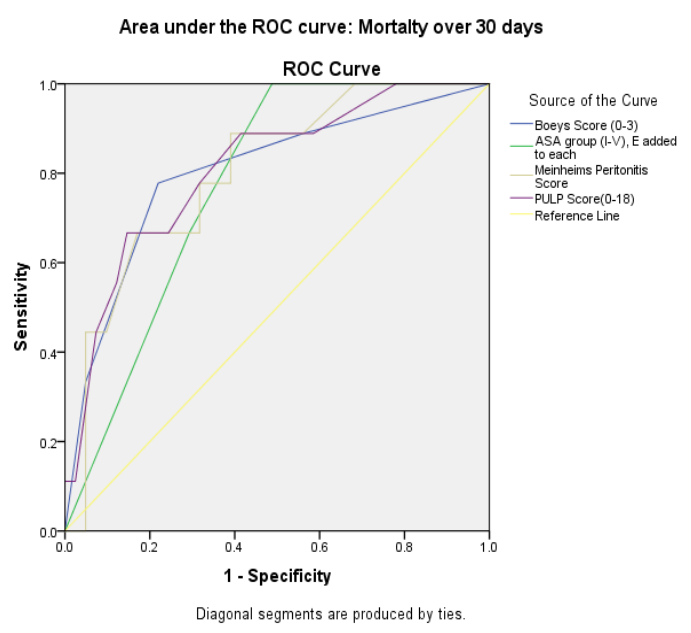

There were significantly higher mean $\pm S \boldsymbol{D}$ of Boey score between patients with 30-day mortality $(2.00 \pm 1.00)$ and those who survived $(0.83 \pm 0.89)$ with $\mathrm{p}$ value $(0.008)$. Similarly mean $\pm S \boldsymbol{D}$ of Boey score were significantly higher in patients who developed postoperative complications within 30 days $(1.41 \pm 1.04)$ and those who didn't develop complications $(0.39 \pm$ $0.50)$ with $\mathrm{p}$ value $(<0.001)$. In following graph (Fig.1), predictability of mortality by different scoring systems has been illustrated.

\section{DISCUSSION}

Mortality and morbidity have been reduced in PPU by the implementation of a standardized evidence-based in hospital care protocol (18). However, in circumstances of a limited number of ICU, high dependency unit beds and large number of referral from all over the country as in our hospital set up, individual risk stratification is of prime importance. Early and accurate identification of patients with increased risk of adverse outcome is needed to plan and target the level of perioperative monitoring and treatment. Thus, a clinical scoring system able to predict the adverse outcome with a high degree of precision is of paramount importance in a resource limited setting like ours. Ideally, score should be easy to calculate, preferably bedside and preoperatively. Boey scoring system uses three parameters: comorbidity, preoperative shock and duration of peptic ulcer perforation all of which can be easily assessed at bedside helping in rapid stratification of serious patients.

Male to female proportion in this study was $11.5: 1.82 \%$ of patients belonged to age $\leq 60$ years. Patients with PUD had other comorbidities in about 11.5- $40.9 \%$ (19). Pulmonary disease was the most frequent comorbid diseases followed by cardiac disease (hypertension) and diabetes mellitus. Only six percentages of our patients had no risk factors. Risk factors in this study were mostly history of regular alcohol consumption, active smoking, history of peptic ulcer disease and long term NSAIDs/steroid intake and previous operation.

Overall 30-day mortality in this study is $18 \%$ which is comparable to $17 \%$ mortality in Buck DL. et al.(20), $16 \%$ in Thorsen K. et al.(21) and $14 \%$ in Makela JT et al. (9) Arici C. et al. (6) each. However, it is higher compared to some other studies $(6-9 \%)(8,12,22-24)$ and is lower than different studies $(20-28 \%)(13,21,25$, 26). First, ours being the tertiary referral center, 
we receive high volume of cases from all over Nepal, most of them often presenting late to us with delayed diagnosis and severe complications.

Second, poor resuscitative efforts at previous hospitals and during patient transport may be responsible for increased mortality. Third, limited ICU facilities, lack of trained critical care team and perioperative standard technology may have compounded further problems. Also, sub-average nutritional status imposes delayed recovery and subsequent morbidity. Lastly many preexisting comorbid conditions undiagnosed previously may be responsible for underestimation of our risk scoring and lead to overall increased morbidity and mortality.

Regarding the causes of mortality, ARDS was the leading cause in this study. More specifically mortality rate in our study is $5.3 \%$, $6.7 \%, 36.4 \%$ and $60 \%$ with Boey score of patients $0,1,2$ and 3 (p-value $=0.08$ ). Improved mortality rate with higher Boey score in our study could be attributed to the improved surgical care in past three decades since Boey first published his study.

In the present study, the morbidity rate of PPU patients was $64 \%$ in which wound infections/ dehiscence, pneumonia/ARDS, and fever were the leading postoperative complications. These findings could be explained by the fact that surgery for PPU is regarded as a contaminated or dirty. Also, chances of nosocomial infections are expected in our hospital setting as compared to different other studies conducted in western and advanced hospital set up. Overall, the postoperative complication rate in the literature ranges from $17 \%$ to $63 \%$ (4). More specifically, risk of morbidity in our study was found to be $42 \%, 53 \%, 100 \%$ and $100 \%$ with Boey score of $0,1,2$ and 3 respectively (p-value - 0.03 ) while in similar study by Lohsiriwat $\mathrm{V}$. et al.(12) the morbidity rates were $11 \%, 47 \%, 75 \%$ and $77 \%$ with Boey scores of $0,1,2$, and 3 respectively $(\mathrm{p}<0.001)$.

We found higher clinical scores (mean \pm S.D) for patients with positive outcomes (mortality or morbidity) as compared to patients without outcomes for all three scores which all were statistically significant. In this study, AUC of Boey scoring in terms of mortality prediction was 0.802 while 0.78 for morbidity.
Several scoring systems have been used to predict mortality and morbidity after PPU surgery. The present study revealed that Boey score, ASA, MPI, and PULP scoring all were capable of predicting poor surgical outcomes, especially Boey score, MPI scoring and PULP score was better than ASA in predicting postoperative mortality. Though MPI is not specifically designed for evaluating PPU cases, it predicts both mortality and morbidity nearly as accurate as Boey and PULP score. However major drawback of MPI is that it requires intraoperative information; therefore, MPI may not be an ideal preoperative scoring system to identify high-risk patients who might need intensive care prior to the operation. Interestingly, ASA score had best morbidity predictor out of all scoring system in this present study. PULP scoring requires use of laboratory investigations.

\subsection{Limitations}

The major limitation of this study is small sample size of just 50 patients due to time constraints and difficulties in patients' follow up. Also, patient nutritional status and BMI are not taken into account which might influence postoperative complications. Laparoscopic surgeries though popular worldwide for PPU, not being done in our set up till now. All surgical procedures were performed by open laparotomy by surgical residents/ registrars under guidance of experienced surgeon. Also, being non-interventional study and follow-up biases are other limitations.

\section{CONCLUSiON}

Boey scoring system has better accuracy than other scoring systems. It is simple, clinically relevant and can precisely predict postoperative morbidity and mortality. Further, it can be calculated at bedside preoperatively and can assist in risk stratification of patients with perforated peptic ulcer. In limited resource setting like ours, this can have various implications: it can help us identify high-risk patients preoperatively and help in better use of limited facilities; risk of adverse outcomes can be explained as part of patient counseling, help preoperative optimization In ICU and extensive perioperative care for overall better outcomes. 


\section{REFERENCES}

[1] Bertleff MJ, Lange JF. Perforated peptic ulcer disease: a review of history and treatment. Digestive surgery. 2010; 27(3):161-9.

[2] Møller MH, Adamsen S, Wøjdemann M, Møller AM. Perforated peptic ulcer: how to improve outcome? Scandinavian journal of gastroenterology. 2009; 44(1):15-22.

[3] Møller MH, Shah K, Bendix J, Jensen AG, Zimmermann-Nielsen E, Adamsen $\mathrm{S}$, et al. Risk factors in patients surgically treated for peptic ulcer perforation. Scandinavian journal of gastroenterology. 2009; 44(2):145-52.

[4] Di Saverio S, Bassi M, Smerieri N, Masetti M, Ferrara F, Fabbri C, et al. Diagnosis and treatment of perforated or bleeding peptic ulcers: 2013 WSES position paper. World Journal of Emergency Surgery. 2014;9(1):45.

[5] Søreide K, Thorsen K, Harrison EM, Bingener J, Møller MH, Ohene-Yeboah M, et al. Perforated peptic ulcer. The Lancet. 2015;386(10000):1288-98.

[6] Arici C, Mesci A, Dincer D, Dinckan A, Colak T. Analysis of risk factors predicting (affecting) mortality and morbidity of peptic ulcer perforations. International surgery. 2006;92(3):147-54.

[7] Boey J, Choi S, Poon A, Alagaratnam T. Risk stratification in perforated duodenal ulcers. A prospective validation of predictive factors. Annals of surgery. 1987;205(1):22.

[8] Lee FYJ, Leung KL, Lai BSP, Ng SSM, Dexter S, Lau WY. Predicting mortality and morbidity of patients operated on for perforated peptic ulcers. Archives of Surgery. 2001;136(1):90-3.

[9] Mäkelä JT, Kiviniemi H, Ohtonen P, Laitinen SO. Factors that predict morbidity and mortality in patients with perforated peptic ulcers. The European journal of surgery. 2002;168(8-9):446-51.

[10] Irvin T. Mortality and perforated peptic ulcer: a case for risk stratification in elderly patients. British journal of surgery. 1989;76(3):215-8.

[11] Chandra SS, Kumar SS. Definitive or conservative surgery for perforated gastric ulcer?-An unresolved problem. International Journal of Surgery. 2009;7(2):136-9.

[12] Lohsiriwat V, Prapasrivorakul S, Lohsiriwat D. Perforated peptic ulcer: clinical presentation, surgical outcomes, and the accuracy of the Boey scoring system in predicting postoperative morbidity and mortality. World journal of surgery. 2009;33(1):80-5.

[13] Møller M, Engebjerg MC, Adamsen S, Bendix J, Thomsen R. The Peptic Ulcer Perforation
(PULP) score: a predictor of mortality following peptic ulcer perforation. A cohort study. Acta Anaesthesiologica Scandinavica. 2012;56(5):655-62.

[14] Daabiss M. American Society of Anaesthesiologists physical status classification. Indian journal of anaesthesia. 2011;55(2):111.

[15] Fitz-Henry J. The ASA classification and perioperative risk. The Annals of The Royal College of Surgeons of England. 2011; 93(3):185-7.

[16] Billing A, Fröhlich D. Prediction of outcome using the Mannheim peritonitis index in 2003 patients. British journal of surgery. 1994; 81(2):209-13.

[17] Linder M, Wacha H, Feldmann U, Wesch G, Streifensand R, Gundlach E. The Mannheim peritonitis index. An instrument for the intraoperative prognosis of peritonitis. Der Chirurg; Zeitschrift fur alle Gebiete der operativen Medizen. 1987; 58(2):84-92.

[18] Møller M, Adamsen S, Thomsen R, Møller A. Multicentre trial of a perioperative protocol to reduce mortality in patients with peptic ulcer perforation. British Journal of Surgery. 2011; 98(6):802-10.

[19] Kim J-M, Jeong S-H, Lee Y-J, Park S-T, Choi $\mathrm{S}-\mathrm{K}$, Hong S-C, et al. Analysis of risk factors for postoperative morbidity in perforated peptic ulcer. Journal of gastric cancer. 2012; 12(1):2635 .

[20] Buck DL, Vester-Andersen M, Møller MH. Accuracy of clinical prediction rules in peptic ulcer perforation: an observational study. Scandinavian journal of gastroenterology. 2012; 47(1):28-35.

[21] Thorsen K, Glomsaker TB, von Meer A, Søreide K, Søreide JA. Trends in diagnosis and surgical management of patients with perforated peptic ulcer. Journal of Gastrointestinal Surgery. 2011; 15(8):1329-35.

[22] Christensen S, Riis A, Nørgaard M, Sørensen HT, Thomsen RW. Short-term mortality after perforated or bleeding peptic ulcer among elderly patients: a population-based cohort study. BMC geriatrics. 2007; 7(1):8.

[23] Egberts J-H, Summa B, Schulz U, Schafmayer C, Hinz S, Tepel J. Impact of preoperative physiological risk profile on postoperative morbidity and mortality after emergency operation of complicated peptic ulcer disease. World journal of surgery. 2007; 31(7):1449-57. 
[24] Subedi SK, Afaq A, Adhikary S, Niraula S, Agrawal C. Factors influencing mortality in perforated duodenal ulcer following emergency surgical repair. JNMA; journal of the Nepal Medical Association. 2007; 46(165):31.

[25] Forsmo H, Glomsaker T, Vandvik P. Perforated peptic ulcer--a 12-year material. Tidsskrift for den Norske laegeforening: tidsskrift for praktisk medicin, ny raekke. 2005; 125(13):1822-4.

[26] Larkin J, Bourke M, Muhammed A, Waldron R, Barry K, Eustace P. Mortality in perforated duodenal ulcer depends upon pre-operative risk: a retrospective 10-year study. Irish journal of medical science. 2010;179(4):545-9.

Citation: Sah Dhruba Narayan. Boey Score in Predicting Outcome in Perforated Peptic Ulcer from Tertiary Referral Center of Nepal. ARC Journal of Surgery.2019;5(1):9-14. doi:dx.doi.org/10.20431/2455-572X. 0501002.

Copyright: (c) 2019 Authors. This is an open-access article distributed under the terms of the Creative Commons Attribution License, which permits unrestricted use, distribution, and reproduction in any medium, provided the original author and source are credited. 